\section{Lassen sich die unterschiedlichen klinischen Verläufe eines früh erkannten Prostatakarzinoms genetisch erklären?}

\author{
Auf den ersten Blick scheint es unmöglich, den Krankheitsverlauf eines Prosta- \\ takarzinoms sicher vorherzusagen. Zu klein sind die untersuchten Patienten- \\ kollektive und zu teuer die Sequenzierungsverfahren. In naher Zukunft \\ scheinen dennoch erste Prognosen möglich.
}

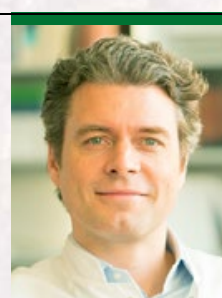

Diese Frage beantwortet für Sie

Prof. Dr. med. Thorsten Schlomm

Martini-Klinik, Prostatakarzinomzentrum

Universitätsklinikum Hamburg-

Eppendorf

Martinistraße 52

20246 Hamburg

E-Mail: tschlomm@uke.uni-hamburg.de
K inisch unterscheidet sich das Prostatakarzinom von anderen soliden Tumoren durch seinen oft langsamen und klinisch indolenten Verlauf. Die Herausforderung der nächsten Jahre wird sein, eine zuverlässige molekulare Klassifizierung des Prostatakarzinoms zu entwickeln, die uns erlaubt, den natürlichen Krankheitsverlauf anhand diagnostischer Stanzbiopsien sicher vorherzusagen. Eine der wichtigsten Aufgaben ist es, zu verstehen, ob unterschiedliche Entstehungs- und Progressionsmechanismen existieren: Gibt es zum Beispiel primär indolente Tumoren, die immer indolent bleiben, oder wird jeder Tumor mit der Zeit aggressiv? Entstehen aggressive Tumore vielleicht auch direkt als solche? Diese Mechanismen zu entschlüsseln ist, wie auch bessere Prognosemodelle zu entwickeln, wichtig, um neue Therapiemöglichkeiten für das metastasierte Prostatakarzinom zu finden.

\section{Häufige genomische Veränderungen identifiziert}

In den letzten Jahren haben wir mithilfe moderner genomweiter Sequenzierverfahren ein immer differenzierteres Bild der molekularen Grundlagen des Prostatakarzinoms erlangen können. Es zeichnet sich ab, dass durch die Kombination klassischer, histologischer und validierter molekularer Marker die Prognoseabschätzung beim Prostatakarzinom verbessert werden kann. Dadurch würde die Active Surveillance möglicherweise in Zukunft für mehr Patienten eine realistische Therapieoption darstellen. Weiterhin lassen sich bei vielen Prostatakarzinomen molekulare Veränderungen entdecken, die darauf hinweisen, dass zielgerichtete molekulare Therapien beim metastasierten Prostatakarzinom wirksam sein könnten.

\section{Übersetzung in die Klinik steht noch aus}

Wenn man den bisherigen Stand der Forschung jedoch kritisch zusammenfasst, muss man sagen, dass wir in den letzten Jahren zwar eine Menge über die molekularen Zusammenhänge und Biologie des Prostatakarzinoms gelernt haben, der wichtigste Schritt jedoch noch fehlt - diese Erkenntnisse in klinisch sinnvolle Tests und Therapien umzusetzen. Das liegt daran, dass gerade die modernen Sequenzierverfahren auf prospektiv gewonnenes Frischgewebe angewiesen sind, für das es aber noch keine ausreichend langen Verlaufsdaten gibt. Zudem gelang es aufgrund der aufwendigen und teuren Sequenziermethoden bisher nur wenige hundert Tumoren komplett auf allen genomischen Ebenen zu untersuchen. Um genetische Marker oder Markerpannel sinnvoll klinisch zu validieren, müssen jedoch tausende Patienten mit dokumentiertem Langzeitverlauf untersucht werden. Das stellt uns derzeit vor zwei relevante He- rausforderungen: Erstens brauchen wir große Patientenkohorten mit dokumentierten klinisch relevanten Endpunkten und zweitens müssen die molekularen Methoden reproduzierbar an teilweise sehr heterogen prozessiertem Paraffingewebe durchführbar sein. Das zweite Problem scheint in naher $\mathrm{Zu}$ kunft gelöst, da neue Sequenziermethoden entwickelt werden.

\section{Große Patientenkollektive fehlen}

Die größte Herausforderung besteht jedoch darin, Patienten mit relevanten klinischen Endpunkten (z. B. klinisch progressionsfreies Überleben ohne Therapie, Entwicklung von Metastasen, karzinomspezifisches Überleben, Therapieansprechen) zu identifizieren. Ausreichend große Kohorten mit solchen Endpunkten stehen jedoch zurzeit nicht zur Verfügung, da der onkologische Langzeitverlauf der meisten Krebspatienten nicht systematisch erfasst wird. Eine globale Prostatakrebsdatenbank zu etablieren könnte die Lösung dafür sein. Bei geschätzten elf Millionen weltweit lebenden und weiteren 20-30 Millionen innerhalb der letzten 20 Jahre verstorbenen Prostatakarzinompatienten muss es das Ziel der Prostatakarzinomgemeinschaft sein, die individuellen Krankheitsverläufe vieler dieser Patienten systematisch zu erfassen und der Wissenschaft zur Verfügung zu stellen.

\section{Globales Prostatakarzinomnetzwerk kann Forschung unterstützen}

Derzeit arbeitet eine internationale Gruppe von Urologen, Patienten und Informatikern gemeinsam an einem globalen Prostatakarzinomnetzwerk. Damit sollen Patienten eine Plattform erhalten, um selber ihre individuellen Tumordaten und Krankheitsverläufe in ein internetbasiertes System einzugeben, sich mit anderen Patienten zu vergleichen, $\mathrm{zu}$ kommunizieren und $\mathrm{zu}$ lernen, wie andere Patienten in der gleichen Situation gehandelt haben. Die Daten stellen wiederum eine wichtige Ressource für die Wissenschaft dar. Es ist zu erwarten, das eine solche Plattform die Forschung massiv unterstützen kann: Bei einer genügend hohen Anzahl von beteiligten Patienten könnten auch Gruppen von Patienten mit seltenen, aber klinisch relevanten Endpunkten in genügend hoher Anzahl identifiziert werden.

Zusammenfassend ist eine sichere Vorhersage des natürlichen Krankheitsverlaufes aufgrund der bisher fehlenden Validierungen an großen Patientenkollektiven mit sinnvollen klinischen Endpunkten bisher noch nicht möglich. Jüngste Entwicklungen zur Etablierung einer globalen Prostatakarzinomdatenbank und neuer Sequenzierverfahren machen jedoch Hoffnung diesem Ziel in naher Zukunft einen großen Schritt näher zu kommen. 